\title{
Radio-iodine therapy of Graves' disease: is there a dose-effect relationship?
}

\author{
David Broggio ${ }^{1,1}$ \\ ${ }^{1}$ IRSN, IRSN/SDOS/LEDI, BP-17, 92626 Fontenay-aux-Roses, France
}

\section{Introduction}

Graves' disease is a benign disease very often treated with radioiodine [1], its incidence is about 30/100 000/year in Europe and USA [2]. Even if several millions of radioiodine treatments have been administrated [1] there are still debates on the exact amount of iodine that should be administrated and on the final goal of the therapy: permanently hypothyroid or euthyroid [3-4].

Inspection of the literature shows that there are little evidences for choosing the requested thyroid dose. In order to obtain the dose-effect relationship for the success of iodine-131 therapy for Grave's disease we carried out an analysis of the literature.

\section{Materials and Methods}

We looked for literature data reporting the success of a single I-131 therapy for Grave's disease. We only included peer-reviewed articles in French or German where the thyroid dose was reported along with the success rate.

We assigned an uncertainty to the success rate (exact Cloper-Pearson method) and to the reported dose. We finally carried out a fit of the data using, as an ansatz, a sigmoid function. In the fitting procedure both error bars were taken into account following the method of Wolberg [5].

\section{Results and discussion}

We extracted from the literature 22 relevant papers accounting for a total of 3922 patients. The reported thyroid doses were between 60 and 649 Gy. In all studies the thyroid dose was calculated with at least one uptake measurement and an ultrasound assessment of the thyroid weight. In 15 of the studies the dose uncertainty was reported or could be deduced. An average dose uncertainty of $26 \%$ was fixed for the other studies.

The resulting fitted dose-effect relationship is shown in figure 1. The data supporting the fit are relatively scattered. However, the fitting procedure naturally produces and average trend and the associated $95 \%$ confidence band. The fitted result is in very good agreement with the 1995 data of Peters [7] which were obtained with 98 patients (data not shown).

\footnotetext{
${ }^{1}$ Corresponding author: david.broggio@irsn.fr
} 
According to our findings the confidence bands for therapy success are as follows: [55$80 \%$ ] at $150 \mathrm{~Gy},[65-90 \%]$ at $200 \mathrm{~Gy}$ and [80-95\%] at $300 \mathrm{~Gy}$.

Several factors can explain the scattered pattern of the obtained data: large uncertainties on axes, different follow-up periods, different regimen of medication withdrawal before therapy. Surprisingly, such a scattered pattern can also be found in multi-centre studies in external radiotherapy.

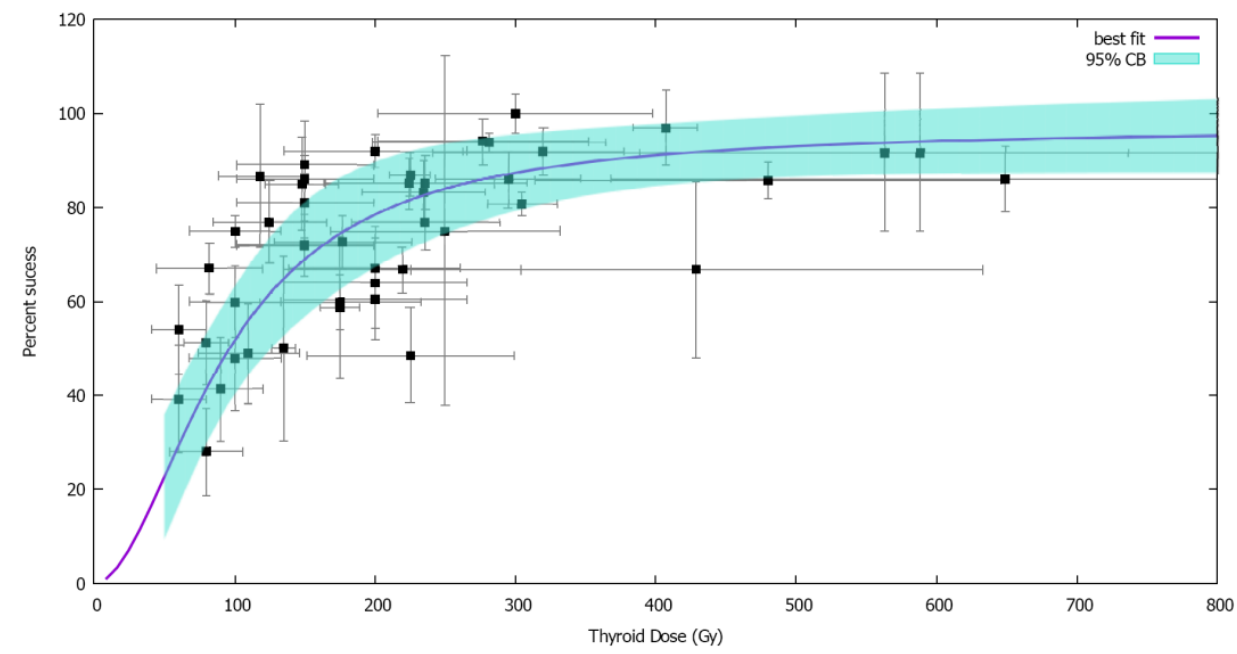

Figure 1. Fitted dose-effect relationship: success rate as a function of thyroid dose.

\section{Conclusion}

To the best of our knowledge this work is the more exhaustive and complete analysis of the dose-effect relationship for one of the most common radio-iodine therapy. On one hand, if one assumes that the data and analysis are reliable it should be concluded that there is no real dose-effect relationship. One the other hand, assuming that a dose effect relationship must exist, it should be concluded that in future studies the dose assessment should be more accurate, using for example several uptake measurements, and that follow-up times and inclusion criteria chosen more rigorously.

\section{References}

1. Bonnema S.J and Hegedüs L. Endocrine Reviews, 33 (6): 920-980 (2012).

2. McLeod D.S.A. and Cooper D.S. Endocrine 42 (2): 252-265 (2012)

3. Van Isselt J.W et al. European Journal of Nuclear Medicine and Molecular Imaging 34 (11):1883-1884 (2007)

4. Kalinyak J.E. and McDougall I.R. Journal of Clinical Endocrinology and Metabolism 88(3):975-977 (2003).

5. Wolberg J. Data Analysis Using the Method of Least Squares, 2006, Springer

6. Peters H. et al. European Journal of Clinical Investigation 25 (3): 186-193 (1995). 